\title{
When a velocitron meets a reflectron
}

A drawback of sector mass spectrometers

(Milestone 1) was the narrow range of mass-tocharge ratio $(\mathrm{m} / \mathrm{z})$ that could be analyzed at any given time. These analyzers could be thought of as 'mass filters', so that acquiring a complete $\mathrm{m} / \mathrm{z}$ spectrum required 'tuning' the filter across all the relevant ranges of interest. This took time, and it was not apt at capturing the spectrum of a sample that either was short-lived or had a composition that changed quickly. Moreover, there were upper limits to the detectable mass that were inherent to the spectrometer.

In 1946, W.E. Stephens from the University of Pennsylvania proposed a new technology to circumvent these limitations. He called it "A pulsed mass spectrometer with time dispersion." The keywords 'pulsed' and 'time dispersion' give away the two main features of this mass analyzer: the use of microsecond pulses of ions, and the fact that ions with different $\mathrm{m} / \mathrm{z}$ reach the detector at different times, allowing ion species to be distinguished by their 'time of flight' (TOF) in the analyzer. The ion pulses are accelerated by an electric field to the same energy and travel down a vacuum tube. Because ions with different $m / z$ have different initial velocities, they will hit the detector at slightly different times, with lighter and/or more charged ions arriving first. In this way, the entire $\mathrm{m} / \mathrm{z}$ spectrum of the sample under study can be

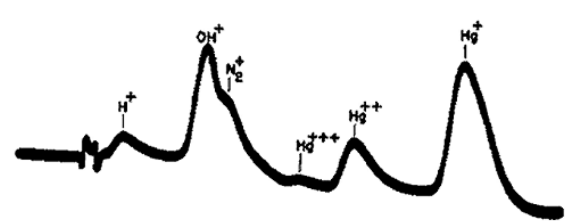

The velocitron developed by Cameron and Eggers could resolve mercury ions with one, two or three positive charges, but not their isotopes. Reprinted with permission from Cameron, A.E. \& Eggers, D.F. Rev. Sci. Instrum. 19, 605-607 (1948). acquired in just hundreds of microseconds, or as long as it takes the heaviest ions to reach the detector. Stephens pinpointed the advantages of his method: "The response time should be limited only by the repetition rate (milliseconds). The indication would be continuous and visual and easily photographed. Magnets and stabilization equipment would be eliminated. Resolution would not be limited by smallness of slits or alignment." The seed of time-of-flight mass spectrometry (TOF-MS) was thus planted.

It took two years before a proof-of-principle TOF spectrometer was developed by A.E. Cameron and D.F. Eggers, then working at Clinton Engineer Works-Tennessee Eastman Corporation. They gave it a more concise name, the 'ion velocitron'. As Stephens had proposed, the velocitron did not need a magnetic field and used ion pulses of 5 microseconds. Cameron and Eggers showed that in this first TOF spectrometer, mercury ions with different charge could be resolved, but their isotopes could not. The poor mass resolution was the result of initial distributions in both energy and spatial location of the ions, which led to dispersions in the measured times not related to $\mathrm{m} / \mathrm{z}$. However, in contrast to previous technologies, the TOF spectrometer did allow mass spectra to be readily visualized on an oscilloscope and updated every millisecond or so.

The mass resolution of TOF was improved by W.C. Wiley and I.H. McLaren in 1955. They devised an improved ion source with two accelerating regions that could correct for the initial ion spatial distribution that is generated by the finite width of the ionization electron beam. A mass resolving power of up to 300 could be achieved in this type of spectrometer, which was significant enough to open the path to commercialization.

In 1973, B.A. Mamyrin and colleagues solved the issue of the initial ion energy distribution. They proposed the use of an electrostatic reflector to detour ions with the same mass but higher velocities, in what they termed a 'reflectron' TOF-MS. Such ions penetrate the electric field to a greater depth, increasing the length of their path toward the detector and thus compensating for the initial differences in energy. The introduction of the reflectron enabled a further increase in mass resolving power, by an order of magnitude, as compared to TOF-MS in which ions propagate linearly.

Introduced commercially in the early 1960s, TOF-MS has seen alternating fortunes, experiencing a renaissance in popularity in the early 1990s due to new methods to produce pulsed ion sources. In particular, the gentle ionization of soft biological macromolecules, whose importance was underlined by the awarding of part of the 2002 Nobel Prize in Chemistry to John Fenn and Koichi Tanaka, for "their development of soft desorption ionisation methods for mass spectrometric analyses of biological macromolecules," spread the application of TOF-MS in the biological sciences. Nowadays, TOF-MS is one of the main mass analyzer technologies available, alongside quadrupoles (Milestone 6), ion traps (Milestone 5) and Fourier transform ion cyclotron resonance (Milestone 5), and is distinguished by a relatively high mass resolving power of up to 60,000 at fast scan speeds.

\section{Elisa De Ranieri, Senior Editor,} Nature Energy

ORIGINAL RESEARCH PAPERS Stephens, W.E. A pulsed mass spectrometer with time dispersion. Phys. Rev. 69, 674792 (1946) | Cameron, A.E. \& Eggers, D.F. An ion "velocitron" Rev. Sci. Instrum 19, 605-607 (1948) | Wiley, W.C. \& McLaren, I.H. Time-of-flight mass spectrometer with improved resolution. Rev. Sci. Instrum. 26, 1150-1157 (1955) | Mamyrin B.A., Karataev, V.I., Shmikk, D.V. \& Zagulin, V.A. The massreflectron, a new nonmagnetic time-of-flight mass spectrometer with high resolution. Sov. Phys. -JETP 37, 45-48 (1973) [Russian original: Zh. Eksp. Teor. Fiz. 64, 82, 1973] FURTHER READING The 2002 Nobel Prize in Chemistryadvanced information (Nobel Media AB, 2014) 